\title{
SÍNDROME DE TAKOTSUBO Y ESTRÉS AGUDO.
}

\author{
Castillo Stalin Bismarck ${ }^{1}$, Pozo Daniela Alejandra ${ }^{2 *}$, \\ Villacís Cecibel Estefanía ${ }^{2}$, Portero María José2.
}

DOI: $10.48018 /$ rmv.v32.i1.S9

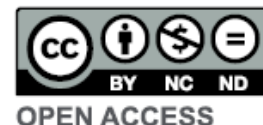

Este artículo está bajo una icencia de Creative Commons de tipo Reconocimien- No comercial - Sin obras derivadas 4.0 International.

1. Hospital Quito $N^{\circ} 1$ de la Policía Nacional. Médico del Servicio de Cardiología. Quito, Ecuador.

2. Hospital Quito $\mathrm{N}^{\circ} 1$ de la Policía Nacional. Médico en funciones hospitalarias. Quito, Ecuador.

\section{ORCID ID:}

Castillo Stalin Bismarck

orcid.org/0000-0001-8040-8935

Pozo Daniela Alejandra

orcid.org/0000-0003-1109-8085

Villacís Cecibel Estefanía

orcid.org/0000-0003-4323-4774

Portero María José

orcid.org/0000-0001-8004-3884

* Corresponding author:

Pozo Daniela Alejandra

E-mail: danielitapozo15@gmail.com

Article history: Manuscript presented at the I Clinical Case Contest - Hospital Vozandes Quito, February 27, 2021.

CARE 2017 Check List statement: The authors have real the CARE 2017 Check List and the manuscript was prepared and revised according to the CARE 2017 Checklist.

Conflict of interest: All authors declared that there are no conflicts of interest.

Financial disclosure: The authors have no financial relationships relevant to this article to disclose.

Forma de citar este artículo: Castillo SB, Pozo DA, Villacís CE, Portero MJ. SÍNDROME DE TAKOTSUBO Y ESTRÉS AGUDO. Rev Med Vozandes. 2021; 32 (1 Suppl 1): S17-S18

\section{Resumen}

Introducción: Síndrome Takotsubo (STk) se caracteriza por una disfunción sistólica regional transitoria en el ventrículo izquierdo, es diagnosticado en el $2 \%$ de los pacientes con clínica de infarto miocárdico con elevación del ST. La etiología es aún incierta postulándose el espasmo arterial epicárdico, alteración microvascular, miocarditis viral, niveles elevados de catecolaminas con alteración del sistema simpático y variaciones anatómicas de la arteria descendente anterior. Se reconocen desencadenantes o gatillos emocionales o físicos para su desarrollo, en ocasiones se presenta en ausencia de ellos. Los síntomas y signos más comunes incluyen: dolor torácico agudo, disnea o síncope, que en un inicio son indistinguibles de un infarto agudo de miocardio. Su presencia varía de acuerdo con el desencadenante: en aquellos con gatillo emocional predomina el dolor torácico y las palpitaciones, mientras que cuando es secundario a estrés físico, las manifestaciones predominantes son las de la enfermedad subyacente (convulsiones, accidente cerebrovascular).

Descripción del Caso: Paciente femenina de 58 años, sin antecedentes cardiovasculares de importancia, quien acude por dolor torácico izquierdo de 2 horas de evolución, asociado aparente estrés emocional. La sospecha clínica inicial fue síndrome coronario agudo. El diagnóstico definitivo se realizó con coronariografía, ventriculografía OAD. Ameritó principalmente anticoagulación con heparinas de bajo peso molecular durante su hospitalización y con nuevos anticoagulantes orales para su manejo ambulatorio, adicional tratamiento para la ansiedad. El seguimiento de la paciente fue desde el mes de octubre del 2019 hasta sus controles ambulatorios posteriores durante el año 2020.

Conclusión: Las características del STK son similares al síndrome coronario agudo (SCA). En mujeres postmenopáusicas. En el caso clínico, cumple 3 de los 4 criterios de Mayo y score de InterTak: 61 puntos. El dolor torácico agudo, disnea o síncope y estudios complementarios pueden confirmar STK. El ECG con elevación del segmento ST (90 \%), onda T negativa en derivaciones precordiales (44\%), onda Q (15-27\%). La elevación del segmento ST en V4-V6 es mayor que en V1-V3, con ausencia de $Q$ anormal. La troponina cardíaca elevada, pero más baja que el IAM; en fase aguda el (BNP o proBNP) podría estar elevado. El aturdimiento miocárdico podría estar mediado por catecolaminas con niveles 2 a 3 veces superiores a un IAM con Killip III. La recuperación no necesita tratamiento, pero puede requerirse el uso de diuréticos, betabloqueadores, inhibidores de la enzima convertidora de angiotensina, bloqueadores de los receptores de la angiotensina-ll, estatinas y ácido acetil salicílico. Tiene pronóstico favorable; mortalidad menor al $2 \%$.

Palabras clave: Síndrome takotsubo, Cardiomiopatía de Takotsubo, Infarto del miocardio, Insuficiencia cardíaca, reporte de caso. 


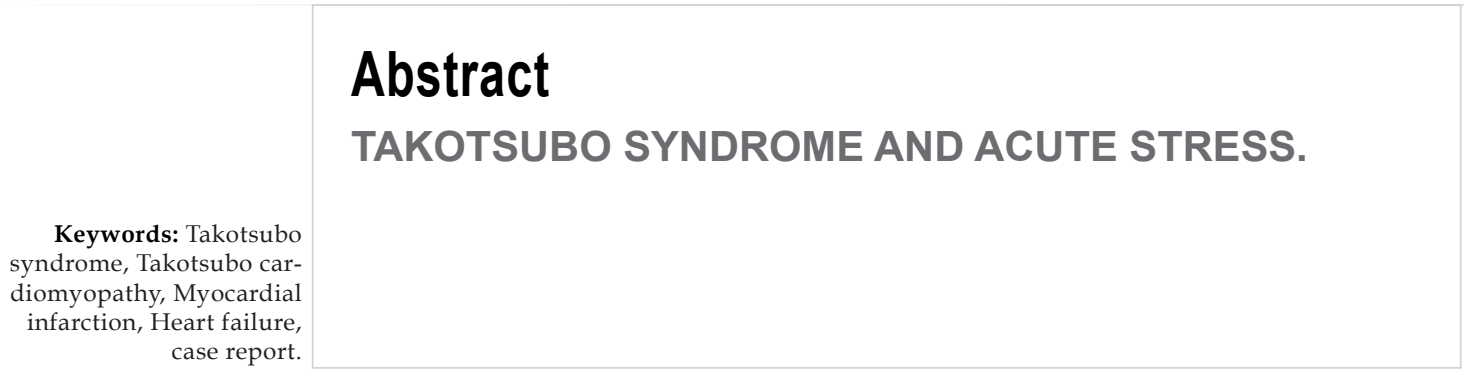

Introduction: Takotsubo Syndrome (STk) is characterized by a transient systolic regional dysfunction on the left ventricle, usually diagnosed in $2 \%$ of the patients presenting with clinical suspicion of ST elevating myocardial infarction (STAMI). Main etiology is still unclear, correlating with pericardial artery spasm, microvascular alterations, viral myocarditis, heightened catecholamine levels with alteration of sympathetic system, and anatomical variations of the anterior descendent artery. Several emotional and physical triggers are linked to its development, but symptoms can arise in their absence. Most common signs and symptoms include: acute chest pain, dyspnea and syncope, initially indistinct to those of an acute myocardial infarction. Its presence varies according to the trigger: on those with a strong emotional trigger chest pain and palpitations, while on physical stress, underlying disease predominates (stroke, seizure).

Case description: A 58 year old female, without medical history of cardiovascular disease, was admitted to the hospital because of left sided chest pain, beginning 2 hours ago, most likely caused by emotional distress. On arrival, initial diagnosis was Acute Coronary Disorder (ACD). Coronarography, and anterior oblique right ventriculography confirming the diagnosis. Requiring mainly low-molecular-weight heparin anticoagulation during admission and novel anticoagulants for outpatient care, added to anxiety treatment. Follow-up started October 2019 and went on during 2020.

Conclusion: STK has similar characteristics to those of ACD on postmenopausal women. The present Clinical Case meets 3 of the 4 Mayo Criteria, and has 61 points on the InterTak score. Acute chest pain, dyspnea and syncope plus several additional studies can confirm STK. EKG showing ST elevation (90\%), negative T wave on precordial leads (44\%), Q wave present (15-27\%). Elevation of the ST segment on V4 to V6 is higher than $V 1$ to $\vee 3$, with absence of $Q$ anomaly. Heightened troponin levels, but lower than AMl; Heghtened BPN or proBPN could be present. Myocardial stunning could be liked to catecholamine levels 2 to 3 times higher than AMI with Killip III. The recovery does not require treatment, but could require diuretics, beta blockers, ACEs, angiotensin-II receptor blockers, statins and acetylsalicylic acid. Prognosis is favorable with mortality under the $2 \%$. 